\title{
Mobility Spectrometer Studies on Hydrazine and Ammonia Detection
}

\author{
William Niu ${ }^{1}$ \\ Hamilton Sundstrand, Pomona, CA, 91767, USA \\ Gary Eiceman ${ }^{2}$ \\ New Mexico State University, Las Cruces, NM 88003, USA \\ Andrew Szumlas ${ }^{3}$ \\ Hamilton Sundstrand, Pomona, CA, 91767, USA \\ and \\ John Lewis ${ }^{4}$ \\ NASA, Houston, TX, 77058, USA
}

\begin{abstract}
An airborne vapor analyzer for detecting sub- to low- parts-per-million (ppm) hydrazine in the presence of higher concentration levels of ammonia has been under development for the Orion program. The detector is based on ambient pressure ionization and ion mobility characterization. The detector encompasses: 1) a membrane inlet to exclude particulate and aerosols from the analyzer inlet; 2) a method to separate hydrazine from ammonia which would otherwise lead to loss of calibration and quantitative accuracy for the hydrazine determination; and 3 ) response and quantitative determinations for both hydrazine and ammonia. Laboratory studies were made to explore some of these features including mobility measurements mindful of power, size, and weight issues. The study recommended the use of a mobility spectrometer of traditional design with a reagent gas and equipped with an inlet transfer line of bonded phase fused silica tube. The inlet transfer line provided gas phase separation of neutrals of ammonia from hydrazine at $50^{\circ} \mathrm{C}$ simplifying significantly the ionization chemistry that underlies response in a mobility spectrometer. Performance of the analyzer was acceptable between ranges of 30 to $80^{\circ} \mathrm{C}$ for both the pre-fractionation column and the drift tube. An inlet comprised of a combined membrane with valve-less injector allowed high speed quantitative determination of ammonia and hydrazine without cross reactivity from common metabolites such as alcohols, esters, and aldehydes. Preliminary test results and some of the design features are discussed.
\end{abstract}

\section{Nomenclature}

$\begin{array}{ll}\text { AM-HY } & =\text { Ammonia Hydrazine } \\ I M S & =\text { Ion Mobility Spectrometer } \\ \text { DMS } & =\text { Differential Mobility Spectrometer } \\ C E V & =\text { Crew Exploration Vehicle } \\ p p b & =\text { Parts-per-billion } \\ p p m & =\text { Parts-per-million } \\ \text { GC } & =\text { Gas Chromatograph } \\ \text { CV } & =\text { Compensation Voltage } \\ \text { RIP } & =\text { Reactant Ion Peak } \\ \text { RF } & =\text { Radio Frequency }\end{array}$

${ }^{1}$ Technical Fellow, Hamilton Sundstrand, 2771 N. Garey Ave., Pomona, CA 91767

${ }^{2}$ Professor, New Mexico State University, Las Cruces, NM 88003

${ }^{3}$ Scientist, Hamilton Sundstrand, 2771 N. Garey Ave., Pomona, CA 91767.

${ }^{4}$ NASA CEV ECLS Manager, NASA-JSC, Houston, TX 77058 


\section{Introduction}

\section{A. Ammonia Hydrazine (AM-HY) Detector Overview}

The Ammonia Hydrazine Detector (AM-HY) is an element of the Orion crew exploration vehicle (CEV). The operating concept of the CEV calls for a parachute return landing into an ocean environment. The plan was to use hydrazine thrusters during the landing maneuver in order to land in the correct orientation. It is possible that upon landing, the fuel systems on-board the CEV could be damaged, leading to a leak of hydrazine fuel into the local environment. In addition, during the initial post-landing period, ammonia evaporation is used as a means to provide cooling for the cabin ${ }^{1}$. The vented ammonia plume may be present in the vicinity of the vehicle for a period of time depending on the local wind condition. The leaked fuel vapor and ammonia coolant present a potential threat to the astronauts inside the CEV when the capsule is necessarily vented to outside air. It has been determined that for astronaut safety, a sensor is necessary to ensure the absence of hydrazine and ammonia from the vicinity of the CEV prior to venting the cabin. To develop this sensor, Hamilton Sundstrand and New Mexico State University collaborateed to apply mobility spectrometer to design an effective, flight-capable sensor for the detection of hydrazine and ammonia at relevant concentrations in the expected environment.

Hydrazine is extremely toxic. Low dosages in the parts-per-billion (ppb) and parts-per-million (ppm) region are hazardous to human health. An ammonia hydrazine detector located in the vicinity of the cabin vent port that can sense both the outside and inside air would be desirable. It should have the function to warn the crew if the external environment is contaminated with hydrazine/ammonia.

In general, ammonia is easier to detect compared to that for the hydrazine thus the effort has been focused on hydrazine detection. An initial trade study has been performed to identify the appropriate sensor for the application. Electrochemical sensors have been used on the ground for detecting hydrazine leaks with some success but would not be able to meet the Orion mission scenario and requirements. Ion mobility devices have been applied for hydrazine monitoring and a handheld version has been modified for a space shuttle experiment ${ }^{2,3}$. Colorimetry and some of the optical methods have also been examined. Considering the Orion mission requirements, the mobility spectrometer is a preferred candidate.

\section{B. Mobility Spectrometers}

Two types of mobility spectrometers have been considered and evaluated. They are the conventional ion mobility spectrometer (IMS) and a variation called differential mobility spectrometer (DMS). IMS is a technique of detecting target compounds by measuring their mobilities, i.e., by measuring their velocities as electrically charged ions in air. The technique is simple in principle. As shown in Figure 1, ions are produced from air molecules by an ionizer such as an electrical spark or a radioactive source. Both positively charged and negatively charged ions are created in the source and, if water is present at ppm or higher concentrations, these typically percolate down to hydrated protons $\left\{\left(\mathrm{H}_{2} \mathrm{O}\right)_{n} \cdot \mathrm{H}^{+}\right\}$and $\mathrm{O}_{2}^{-}$, respectively. These 'reactant ions' can transfer charge to trace contaminants by either proton or electron transfer, or can directly react with contaminants to generate ions. Any ions formed are then drawn through the analyzer under an electric field, timed as they transit a drift region, and the time to their arrival at a detector is converted to mobilities. Because water plays an important part in the ion-molecule chemistry, it is necessary to control the water vapor concentration within the spectrometer; optimum performance is best achieved by operating with relatively dry air containing low ppm levels of water vapor. For this reason, recirculated dry air is supplied to the spectrometer. The technique is attractive due to its high intrinsic sensitivity, its conceptual simplicity and its potential for miniaturization ${ }^{4}$.

Measurement of ion mobilities as a function of time of flight in a drift tube under an applied electric field was developed in the early 70's. For almost 20 years, ion mobility spectrometers have been successfully deployed for detecting chemical warfare agents for military applications and more recently for explosives at airports. It has also been applied for environmental monitoring, usually in conjunction with a gas chromatograph (GC). IMS is highly sensitive and can be made portable.

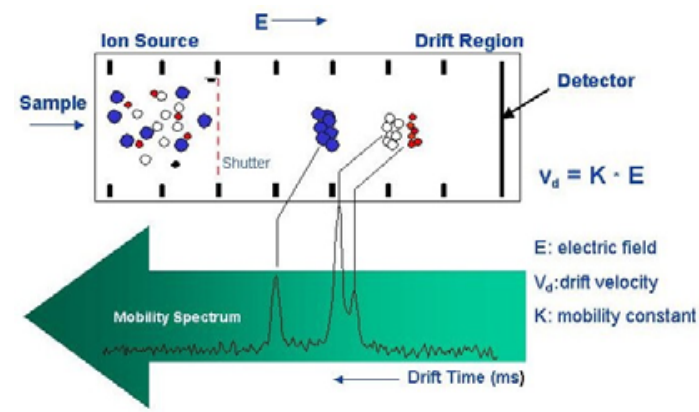

A variation of IMS is called Differential Mobility Spectrometry (DMS), or Field Asymmetric Ion Mobility Spectrometry (FAIMS).

Figure 1. Schematic for a conventional Ion Mobility Spectrometer. Ions arrive at the detector according to their mobilities. 
In DMS, ions are carried through a drift tube while being subjected to an oscillating radio frequency (RF) electric field. The RF field is applied at right angles to the direction of mass flow, and is shaped to pull ions rapidly in one direction and restore them more slowly toward the drift tube center. Depending on how the various ions' mobilities change under the high and low alternating fields, the shaped RF will pull some ions out of the flow, allowing only a narrow range to transmit through the drift tube and reach the detector. In this manner the DMS operates as an RF ion filter, an analog of a quadruple mass spectrometer, in which high sensitivity can be developed by long dwell times for ions of interest when operating in a selected ion mode. The DMS can also be scanned continuously

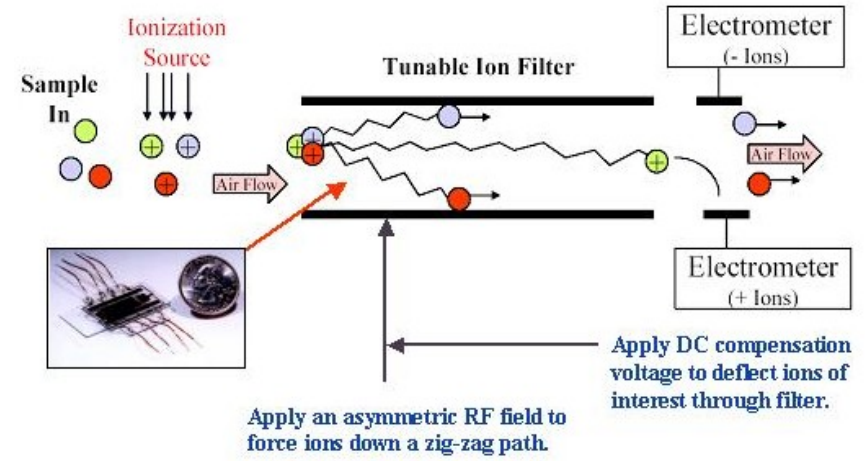

Figure 2.Operation of the DMS. Ions that make it through the filter region have high-field mobilities equal to their low-field mobilities.

across the range of ions present, or can be step-scanned to extract target ions sequentially from a mixture. The entire analyzer is a sandwich constructed of micro-fabricated electrodes, and requires only a flow of sample air to operate.

As ions move through the ion filter, an asymmetric RF field is applied perpendicular to the ion path. The asymmetric RF applies a very high electric field briefly, followed by a lower field in the other direction for a longer time. Any ions formed in the atmosphere pressure ionization source experience this asymmetric field and move with a zigzag motion down the filter. Most ions will hit the plates where they are neutralized and become invisible to the electrometer Faraday collectors. The resulting neutral molecules are carried out of the spectrometer by the gas flow. Only ions whose high-field mobilities exactly equal their low-field mobilities will make it through to the detectors. The filter is tuned to ions of different differential mobilities by applying a separate DC bias on top of the RF field. This DC or 'compensation' field is used to counter a specific target ion's drift toward the walls, restoring ions of a narrow range of differential mobility down the centerline of the drift tube and forcing all others to be neutralized at the walls. Determining the range of ions present is accomplished by scanning the compensation voltage $(\mathrm{CV})$.

Ions created in both types of mobility spectrometers rely on the charge transfer between the reactant ions and the target molecules. Ammonia and hydrazine have relatively high proton affinities thus affording high sensitivity and interference rejection.

\section{Ammonia Hydrazine Detector Design}

Hydrazine is a colorless, oily, flammable liquid from 2 to $113.5^{\circ} \mathrm{C}$. Hydrazine exposure is destructive to both skin and mucous membranes. Its vapor is extremely irritating to the eyes and can cause temporarily blindness. The lungs, liver, kidney and central nerve system may also be affected after exposure ${ }^{5}$. The NASA spacecraft maximum allowable concentrations (SMAC) ${ }^{6}$ for hydrazine are shown in Table I. As another data point, the American National Institute for Occupational Safety and Health (NIOSH) stated that the occupational exposure as defined as the timeweighted average is $0.01 \mathrm{ppm}$, with a ceiling level of $0.03 \mathrm{ppm}$.

Ammonia is also a hazardous gas but its health effect is less severe compared to hydrazine. Ammonia is destructive to tissues of the mucous membrane and upper respiratory tract. The SMAC levels for ammonia are also shown in Table I.

Table I. SMAC levels for Ammonia and Hydrazine.

\begin{tabular}{|r|c|c|c|c|c|}
\hline Chemical & $\begin{array}{c}\mathbf{1} \text { Hour } \\
\text { Exposure }\end{array}$ & $\begin{array}{c}\text { 24 Hour } \\
\text { Exposure }\end{array}$ & $\begin{array}{c}\text { 7 Day } \\
\text { Exposure }\end{array}$ & $\begin{array}{c}\text { 30 Day } \\
\text { Exposure }\end{array}$ & $\begin{array}{c}\text { 180 Day } \\
\text { Exposure }\end{array}$ \\
\hline Hydrazine & $4 \mathrm{ppm}$ & $0.3 \mathrm{ppm}$ & $0.04 \mathrm{ppm}$ & $0.02 \mathrm{ppm}$ & $0.004 \mathrm{ppm}$ \\
\hline Effect & Death & Hepatotoxicity & Hepatotoxicity & $\begin{array}{l}\text { Hepatotoxicity } \\
\text { Liver Hyperplasia } \\
\text { Nose Cancer }\end{array}$ & $\begin{array}{l}\text { Hepatotoxicity } \\
\text { Nose Cancer }\end{array}$ \\
\hline Ammonia & $30 \mathrm{ppm}$ & $20 \mathrm{ppm}$ & $10 \mathrm{ppm}$ & $10 \mathrm{ppm}$ & $10 \mathrm{ppm}$ \\
\hline Effect & Mucosa Irritation & Mucosa Irritation & Mucosa Irritation & Mucosa Irritation & Mucosa Irritation \\
\hline
\end{tabular}




\section{A. Inlet System}

During the sea landing phase, it is possible that sea water may enter the vehicle and the detector unexpectedly. Thus the detector must be robust and insensitive to water. A membrane inlet system has been considered. The concept of using permeable membranes for the separation of gases and vapors has been developed for many years. It is based on the fact that different organic materials permeate through the membrane differently. With proper selection of membrane material, certain compounds can preferentially pass through. For mobility spectrometer application, dimethylsilicone is commonly used because of its high moisture rejection capability. The membrane acts as the inlet to the system. Sample vapor can dissolve in the membrane and diffuse through to the ion source of the analyzer. The membrane provides selectivity to the system and has been successfully used for ion mobility spectrometers ${ }^{7}$.

Several types of membranes have been tested, including methylsilicone membranes from two IMS manufacturers and a custom built membrane from our laboratory. With these membrane inlets, sub-ppm hydrazine can be detected with ion mobility spectrometers. The transmission efficiencies of these membranes are somewhat low and optimization is needed for this particular application.

\section{B. Compound Separation}

Both ammonia and hydrazine may be present in the environment. Since the ammonia is less toxic than that for the hydrazine, the alarm levels will be set differently. It is important to differentiate them so it won't cause unnecessary alarm. For example, $10 \mathrm{ppm}$ ammonia in the cabin would be acceptable but may be deadly if $10 \mathrm{ppm}$ hydrazine is present.

As discussed in Section I, ionization processes in mobility spectrometers relies on charge transfer. Competitive charge exchange between ammonia and hydrazine in the ion source of the mobility spectrometer will affect quantitative determinations, especially when ammonia levels are significantly higher than that of the hydrazine. This is not only because of charge competition but also the possible formation of new ion clusters containing ammonia and hydrazine causing wholly new spectra that are concentration dependent. The detector therefore must provide some degree of separation of these two compounds. The pre-separation could include a membrane, a high-speed chromatographic column and a sample introduction device. To minimize loss

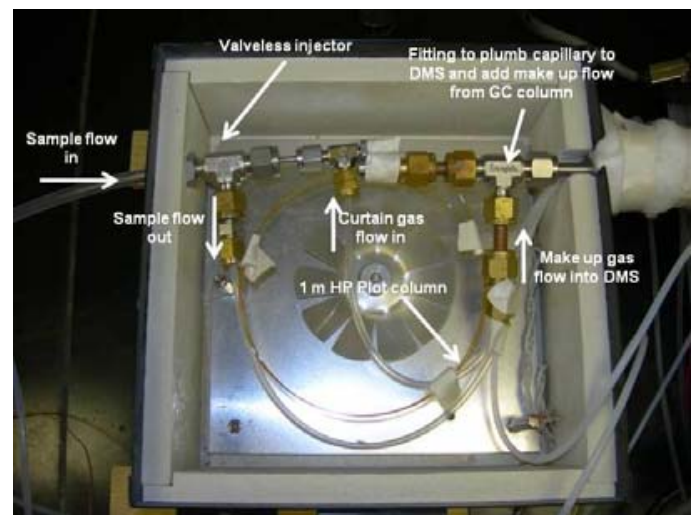

Figure 3. Sample Introduction System. The detector is on the right. at the sample introduction, a valveless injection port has been breadboarded for initial testing, as shown in Figure 3. In this scheme, a sample pump draws ambient sample into the sample inlet assembly. A compressed nitrogen gas (or filtered air) shields the capillary sample line from ambient sample. The compressed gas line is also connected to a vent line. Open the relief valve and the curtain gas recesses causing the sample to get into the capillary inlet, resulting in a sample pulse injecting into the chromatographic column. This sample introduction scheme is similar to the vapor sampling device reported in the literature ${ }^{8}$. This sample introduction system can be packaged into a compact volume attached to the analyzer.

\section{Sensor Response}

In the event a leak is developed while the cabin is opened to the external environment, fast sensor response time will be essential to provide the crew member sufficient time to react. The mobility spectrometer has very fast response time, in seconds for DMS and milliseconds for IMS. The system response time resides in the sample transport and separation. With the breadboard sample introduction system and a short chromatographic column, the response time can be kept under one minute.

Different concentrations of hydrazine and ammonia have been tested ranging from $100 \mathrm{ppb}$ to tens of ppm concentrations. Ammonia mixture can be stored in a gas tank and is relatively easy to handle. A Kintek oven is used to quantitatively generate hydrazine vapors. In this setup, a permeation tube with a known permeation rate is placed inside the oven. The amount of hydrazine permeating through the tube is controlled by the oven temperature. An inert gas, usually nitrogen, is used to carry the known concentration of hydrazine vapor into the sample introduction system of the detector. A separate inert gas stream can be used to dilute the hydrazine containing stream into different concentrations. Hydrazine is very difficult to transport and great care must be exercised in designing the 
sampling system to avoid sample hang-up or contamination. To ensure that the hydrazine vapors are properly generated, the sample was diverted into a mass spectrometer for verification.

\section{Preliminary Test Results}

\section{A. Differential Mobility Spectrometer (DMS)}

A commercial DMS device was used for initial testing. As shown in Figure 4, the Kintek oven on the left generates hydrazine vapor and feeds into the DMS. One of the main parameters in DMS technologies is the separation voltage needed to obtain separation in a DMS since chemistry of ionization for hydrazine was virtually assured owing to the high proton affinity of hydrazine. Another consideration is the appearance of hydrazine at particular compensation voltages (CV) for a certain setting of separation voltage.

Figure 5 shows an overlay of spectra of clean air and hydrazine of $100 \mathrm{ppb}$. The reactant ion peak (RIP) was a hydrated proton with the form $\mathrm{H}^{+}\left(\mathrm{H}_{2} \mathrm{O}\right)_{n}$ where $\mathrm{n}$ is dependent upon moisture in the internal gas atmosphere and gas temperature. The hydrated proton reacts with the hydrazine vapor resulting in a product ion, $\mathrm{N}_{2} \mathrm{H}_{4} \mathrm{H}^{+}\left(\mathrm{H}_{2} \mathrm{O}\right)_{n-1}$. Notably, the product ion is on scale in this figure at an amplitude of $0.55 \mathrm{~V}$ and the RIP is fully depleted here also. While the noise background is less than $0.05 \mathrm{~V}$, this suggests that there is sufficient signal thus the limit of detection should be a factor of 10 less or $10 \mathrm{ppb}$. The high sensitivity provides sufficient margin for transport loss in the sampling system, e.g., a membrane.

In the spectrum for DMS response to ambient air (red line), the hydrated proton is evident in a peak at CV farther from zero volts (the point of reference in analysis of DMS spectra) than that for the product ions of hydrazine, seen in the spectrum for analysis of hydrazine in air (black line). This is consistent with the anticipated field dependences in DMS which scale largely on mass.

In figure 5, the spectrum for hydrazine shows no reactant ion peak telling that the instrument is already saturated in response. In this case, a shorter sample pulse can be used to reduce the effective number of hydrazine molecules entering the sampling system. Essentially, we can control the dynamic range of the instrument.

\section{B. Ion Mobility Spectrometer (IMS)}

A home-made ion mobility spectrometer was used for the initial study. Mobility spectra for ammonia or hydrazine in a room temperature mobility spectrometer are poorly resolved. The separation of ions for ammonia and hydrazine showing in Figure 6 is actually better here than that with a hand-held membrane inlet IMS. This could be attributed to low moisture, high gas purity and slightly warmed drift tube here. Under these conditions, the formation of water clusters is suppressed and resolution of ions nearly by mass is evident with ammonia, hydrated proton, and hydrazine ions at 5.8, 6.3 and $7.5 \mathrm{~ms}$, respectively. The peak shape is poor with resolving power of about 15 with a shutter pulse width of 400 microseconds. The separation of ion peaks in Figure 6 may be sufficient with signal processing to pull quantitative information, but barely sufficient. A hyphenated system of GC-IMS will aid quantitative measurements since retention differences will deliver chemicals separately to the drift tube. Still, the use of hydrated ions at room temperature makes the technology vulnerable to variations in moisture and place high demands on engineering of moisture control. Additionally, the use of the detector for external air and cabin air 
favors the use of a reagent gas to suppress undesirable response from ketones, alcohols, aldehydes, and other metabolic vapors.

To enhance the separation of hydrazine and ammonia in the IMS, the system can be doped with acetone. The reagent gas chemistry to replace water-based cluster reactions is still the best option to stabilize behavior and in conjunction with preseparation, provide quantitative confidence for ammonia and hydrazine in a single instrument and measurement. The chemistry of acetone modification of gas atmospheres for hydrazine detection in mobility spectrometers is fairly well established. The acetone doped system connected to a fast chromatographic separation shows good resolution of ammonia and hydrazine as shown in Figure 7. The y-axis shows the chromatographic separation in seconds and the $\mathrm{x}$-axis depicts the ion drift in mili-seconds.

\section{Mobility Spectrometer Trade Considerations}

As shown in III A and B, both DMS and IMS can be used for the AM-HY detector. There are several parameters affecting the trade between the IMS and DMS. In a purified gas atmosphere, separation of ammonia and hydrazine in an IMS will be poor. In contrast, DMS shows better resolving power with ions of low mass with the current generation of small analyzers considered here. However, even with pre-separation of sample, there is a concern that background interferences will complicate ion chemistry since the pre-separation will be low resolving, just enough to separate ammonia from hydrazine as molecular forms. Thus, matrix interferences may complicate response and use of a reagent gas is advised. In the presence of a reagent gas such as acetone, ammonia and hydrazine will form ion molecule associations making the ions large. In such a circumstance, IMS will resolve large ions better than DMS where high-molecular weight ions will form cluster near zero CV. In terms of robustness in operating at different environments, spectra of both spectrometers are affected by temperature, pressure, and humidity. Nevertheless, IMS spectrum appears to be more stable and is more predictable. Finally, the power consumption of IMS is less than that of the DMS, which needs to support the generation of a high RF voltage. Since power is at a premium during the post-landing phase, at this point, IMS is more favored for the Orion application.

\section{Summary}

Initial feasibility and design for an ammonia hydrazine detector have been performed for the Orion application. Methods and materials were demonstrated in a challenging sensing requirement to detect quantitatively hydrazine in the presence of ammonia with unknown and unpredictable levels, with a response time under one minute. The system consists of a mobility spectrometer of linear field design with temperature between 40 to $60^{\circ} \mathrm{C}$ and an inlet containing a valve-less injector and short section of capillary tubing. The IMS will be based on alternate reagent gas chemistry with acetone or nonanone. The inlet should also be equipped with a membrane and preliminary tests have demonstrated the utility of a membrane inlet. Although speed, limits of detection, and resolving power were demonstrated at subsystem levels, a brassboard system should be developed and undergo rigorous quantitative testing. 


\section{Acknowledgments}

This work was performed under NASA Contract \#NNJ06TA25C.

\section{References}

${ }^{1}$ Lewis, J., Barido R., Cross C., Peterson L., Tuan G., and Carrasquillo R., “Crew Exploration Vehicle Environmental Control and Life Support Development Status.” SAE Technical Paper Series 2008-01-2079, 38th International Conference on Environmental Systems, San Francisco, California, July 2008.

2Eiceman, G., Salazr. M., Rodriguez, M., Limero, T. Beck, S., Cross, J., Young, Y., and James, J., "Ion Mobility of Hydrazine, Monomethylhydrazine, and Ammonia in Air with 5-Nonanone Reagent Gas.” Analytical Chemistry, Vol 65, 1993, pp 1696-1702

${ }^{3}$ Bollan H., Stone, J., Brokenshire, J., Rodriguez, J., and Eiceman, G., "Mobility Resolution and Mass Analysis of Ions from Ammonia and Hydrazine Complexes with Ketones Formed in Air at Ambient Pressure.” J. Am. Soc. Mass Spectrometry, Vol 18, 2007, pp940-951

${ }^{4}$ Eiceman, G., and Karpas, Z., “Ion Mobility Spectrometry” CRC Press, 2005, ISBN 0-8493-2247-2

${ }^{5}$ Makarovsky, I., Markel, J., Dushnitsky, T., and Eisenkraft, A., “Toxic Chemical Compound”, Israel Medical Asso Journal, Vol 10, 2008, pp 302-306

${ }^{6}$ Toxicology Group, Medical Operations Branch, Medical Science Division, Space and Life Sciences Directorate, NASAJSC, “Spacecraft Maximum Allowable Concentration for Airborne Contaminants.” JSC 20584, June 1999

${ }^{7}$ Turner, R., “Transition Analytical Instrumentation from the Laboratory to Harsh Environment.” Pure Appl. Chem., Vol 74, 2002, pp. 2317-2322

${ }^{8}$ Arnold, N., McClennen, W., and Meuzelaar, H., "Vapor Sampling Device for Direct Short Column Gas Chromatography/ Mass Spectrometry Analyses of Atmospheric Vapors.” Analytical Chemistry, Vol. 63, 1991, pp 299-304 